\title{
SARCOMA DE EWING: ASPECTOS CLÍNICOS E RADIOGRÁFICOS DE 226 CASOS*
}

\author{
Julian Catalan ${ }^{1}$, Alexandre Calábria da Fonte ${ }^{1}$, Joel Rodrigo Beal Lusa ${ }^{1}$, Alex Dias de Oliveira ${ }^{1}$, \\ Elisa Soares de Melo ${ }^{1}$, Carlos Marcelo Gonçalves ${ }^{2}$
}

\begin{abstract}
Resumo OBJETIVO: Avaliar os aspectos radiográficos e clínicos presentes em pacientes com o diagnóstico de sarcoma de Ewing confirmado por histopatologia. MATERIAIS E MÉTODOS: Foram analisados, neste trabalho, os dados clínicos e radiográficos (quando disponíveis) de 226 pacientes com o diagnóstico de sarcoma de Ewing ósseo. RESULTADOS: Dos casos avaliados, 61,5\% (139) eram do sexo masculino e 83,7\% (189) eram brancos. A mediana de idade dos pacientes foi de 14 anos e a topografia mais freqüente das lesões foi o osso ilíaco, em $13,7 \%$ (31) dos casos. 0 aspecto radiográfico mais comum foi o de lesão lítica com reação periosteal (padrões variados), em 32,7\% (74) dos casos. CONCLUSÃO: 0 sarcoma de Ewing ósseo é uma neoplasia bastante agressiva, ocorrendo mais comumente em indivíduos na segunda década de vida e cujo aspecto radiográfico mais comum é o de lesão lítica com reação periosteal típica de lesão agressiva. Unitermos: Radiologia convencional; Sarcoma de Ewing; Neoplasias ósseas.
\end{abstract}

Abstract Ewing's sarcoma: clinical and radiographic aspects of 226 cases.

OBJECTIVE: To review the clinical and radiographic findings in patients with histologically confirmed Ewing's sarcoma. MATERIALS AND METHODS: Clinical and radiological (whenever available) data of 226 patients with Ewing's sarcoma of the bone were analyzed. RESULTS: Of the evaluated cases, $61.5 \%$ (139) were male and $83.7 \%$ (189) were white. The average age was 14 years old and the most common site of the lesions was the iliac bone, seen in $13.7 \%$ (31) of the cases. The most common radiological findings were Iytic lesion with periosteal reaction, seen in $32.7 \%(74)$ of the patients. CONCLUSION: Ewing's sarcoma of bone is an aggressive neoplasm, occurring mainly in the second decade of life, which main radiological findings are lytic lesions with periosteal reaction, typical characteristic of aggressive lesions.

Key words: Conventional radiology; Ewing's sarcoma; Bone neoplasms.

\section{INTRODUÇÃO}

O sarcoma de Ewing é uma neoplasia óssea de comportamento biológico bastante agressivo, que acomete principalmente indivíduos abaixo dos 30 anos de idade e apresenta discreto predomínio em pacientes do sexo masculino. Pode surgir em qualquer osso do corpo, predominando nas extremidades e pelve, além de existir uma variante que surge em partes moles e é histologicamente indistinguível.

Nos ossos tubulares, várias entidades são similares com relação a seu aspecto radiográfico, porém, diante de uma lesão os-

* Trabalho realizado no Centro de Tratamento e Pesquisa Hospital do Câncer - A.C. Camargo, São Paulo, SP.

1. Médicos Residentes do Departamento de Imagem do Centro de Tratamento e Pesquisa Hospital do Câncer - A.C. Camargo.

2. Médico Radiologista do Departamento de Imagem do Centro de Tratamento e Pesquisa Hospital do Câncer - A.C. Camargo.

Endereço para correspondência: Dr. Julian Catalan. Rua Urano, 1, ap. 75, Aclimação. São Paulo, SP, 01529-010. E-mail: juliancatalan@uol.com.br

Recebido para publicação em 28/10/2004. Aceito, após revisão, em 12/2/2005. teolítica, permeativa, com reação periosteal laminada e interrompida, dando origem aos denominados "triângulos de Codman", e com grande aumento das partes moles associado, deve-se aventar esta possibilidade diagnóstica.

Com a evolução dos agentes quimioterápicos, a sobrevida e a chance de cura dos pacientes com este tumor têm melhorado consideravelmente nos últimos anos.

\section{MATERIAIS E MÉTODOS}

Foram analisados, neste trabalho, 226 casos de sarcoma de Ewing diagnosticados entre 1980 e 2000 em nossa instituição e histologicamente confirmados, através de dados colhidos em prontuários e de revisão do arquivo de imagens do setor de radiologia.

Não houve padronização na realização dos exames, por terem sido efetuados na rotina do serviço ao longo dos anos, em diferentes aparelhos, sem definição de protocolo próprio.

\section{RESULTADOS}

Dos 226 casos avaliados, $61,5 \%$ (139) eram do sexo masculino e 38,5\% (87) eram do sexo feminino; $83,7 \%$ (189) eram brancos, $12 \%$ (27) eram pardos, $3 \%$ (sete) eram negros e 1,3\% (três) eram amarelos. A média de idade dos pacientes foi de 14 anos, com 56,6\% (128) deles na segunda década de vida (Figura 1). Os sintomas mais freqüentes foram dor, presente em $79,6 \%$ (180) dos pacientes, e aumento de volume

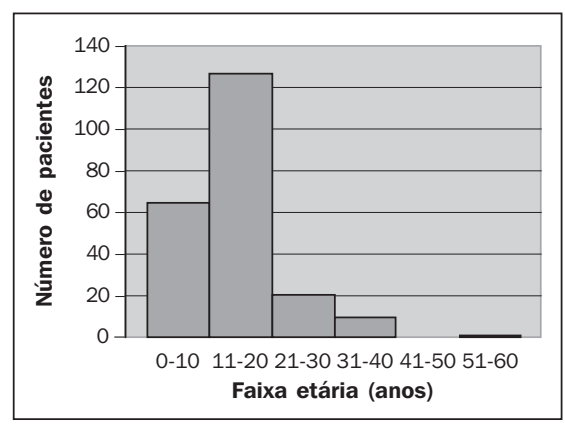

Figura 1. Gráfico demonstrando a distribuição dos pacientes com relação à faixa etária. 


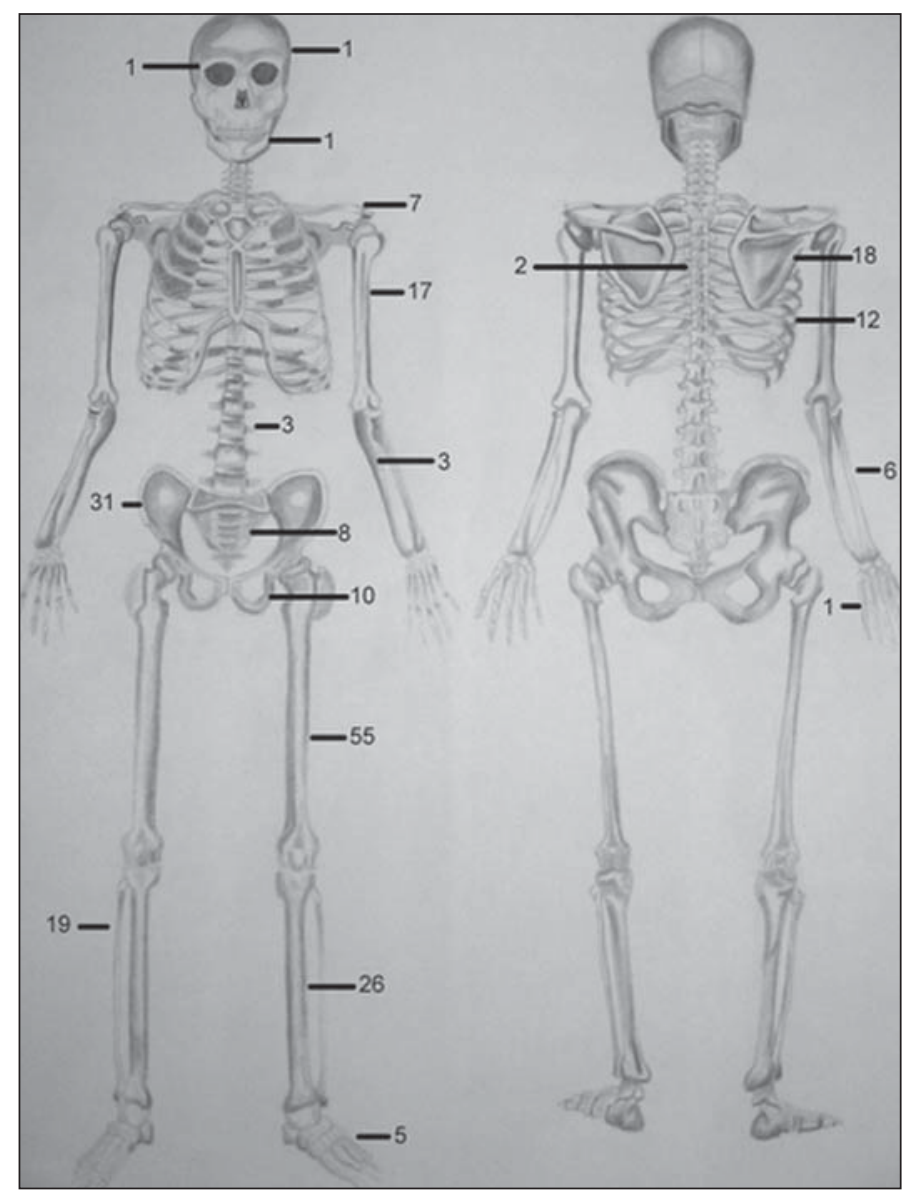

Figura 2. Desenho esquemático do esqueleto demonstrando a distribuição topográfica das lesões.

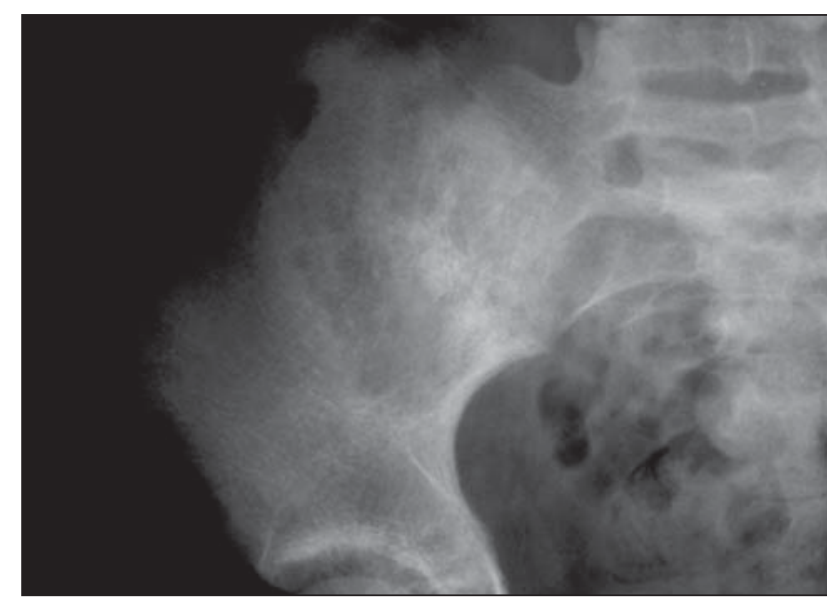

Figura 3. Radiografia pélvica demonstrando lesão mista, de limites mal definidos, no osso ilíaco direito.

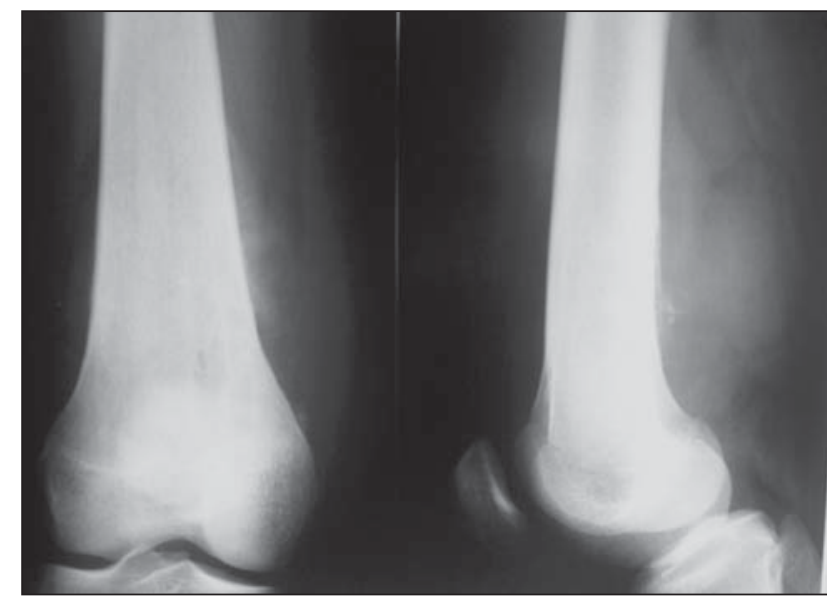

Figura 4. Sarcoma de Ewing. Radiografia do fêmur distal com lesão esclerótica metafisária e reação periosteal espiculada (em "raios de sol"). Tanto o padrão radiográfico da lesão quanto da reação periosteal são mais característicos de um osteossarcoma. local, em 52,2\% (118). Emagrecimento foi relatado por $12 \%$ (27) dos pacientes e febre foi referida por $6,6 \%$ (15). Fratura patológica foi o sintoma inicial em apenas $0,9 \%$ (dois) dos pacientes. O tempo médio entre o início dos sintomas e o diagnóstico foi de oito meses.

A topografia mais frequiente das lesões (Figura 2) foi o osso ilíaco (Figura 3), com $13,7 \%$ (31 casos), em seguida a metáfise distal do fêmur (Figura 4), com 8,4\% (19 casos), escápula (Figura 5), diáfise femoral (Figura 6) e metáfise proximal do fêmur (Figura 7), com 8\% (18 casos), metáfise proximal da tíbia com $6,6 \%$ (15 casos), arcos costais com 5,3\% (12 casos) e metáfise proximal do úmero e ísquio-púbis em $4,4 \%$ (dez casos).

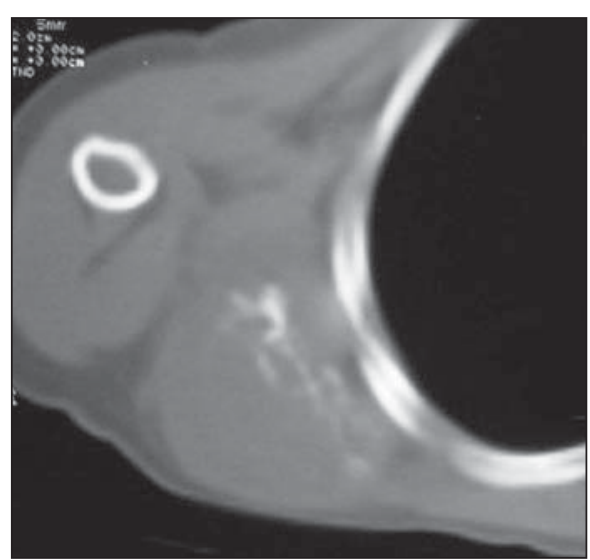

A

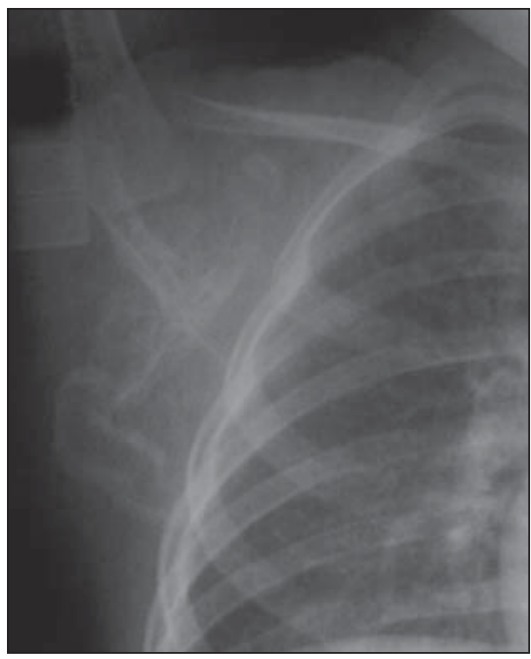

B

Figura 5. Sarcoma de Ewing da escápula. Radiografia (A) e tomografia computadorizada (B) mostrando destruição óssea por lesão lítica com grande componente de partes moles associado. 


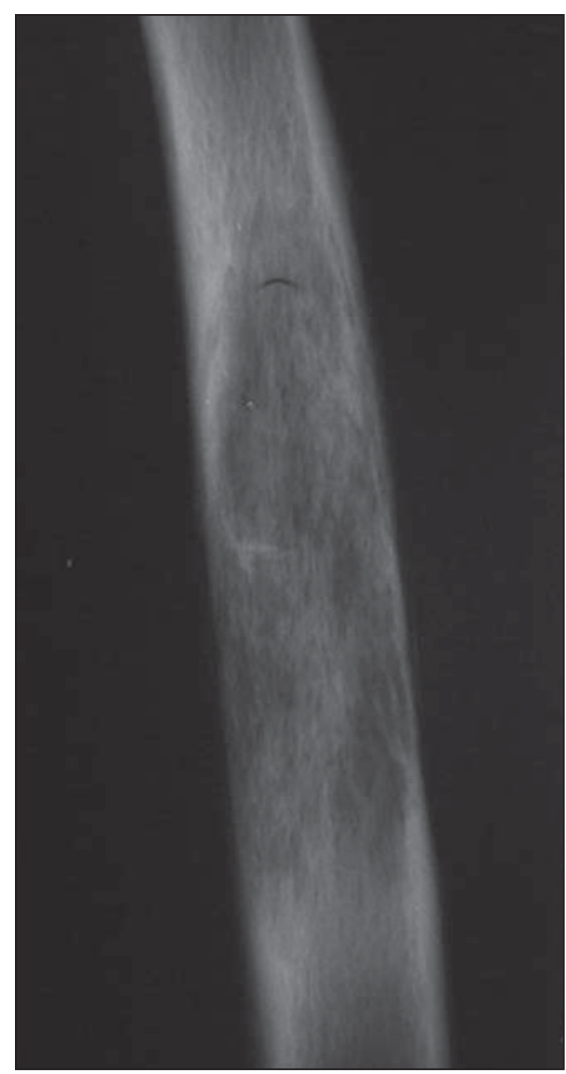

Figura 6. Sarcoma de Ewing do fêmur. Lesão diafisária lítico-permeativa (roído de traça), com limites mal definidos.

Dos 190 pacientes em que não houve perda de seguimento, 36,3\% (69) encontravam-se vivos durante o levantamento de dados e 63,7\% (121) evoluíram para óbito. Destes, a sobrevida média foi de dois anos e dois meses. $\mathrm{O}$ fato de ser uma instituição de referência em oncologia, para onde muitos pacientes com poucas opções terapêuticas e em avançado estágio de evolução da doença são encaminhados, além de muitos dos casos serem antigos, do início da década de 80 , justifica a curta sobrevida observada nesta série.

Os órgãos mais acometidos por metástases foram o pulmão, em 36,2\% (82) dos pacientes, ossos em $30 \%$ (68), fígado em $5,3 \%$ (12) e linfonodos em apenas $1,3 \%$ (três) dos pacientes.

Os aspectos radiográficos mais freqüentemente encontrados foram os de lesão lítica com reação periosteal (vários padrões diferentes) em 32,7\% (74) dos pacientes, lesão lítica pura em 18,1\% (41), lesão mista pura em $6,2 \%$ (14) e lesão permeativa em $5,3 \%$ (12) dos pacientes. Não havia

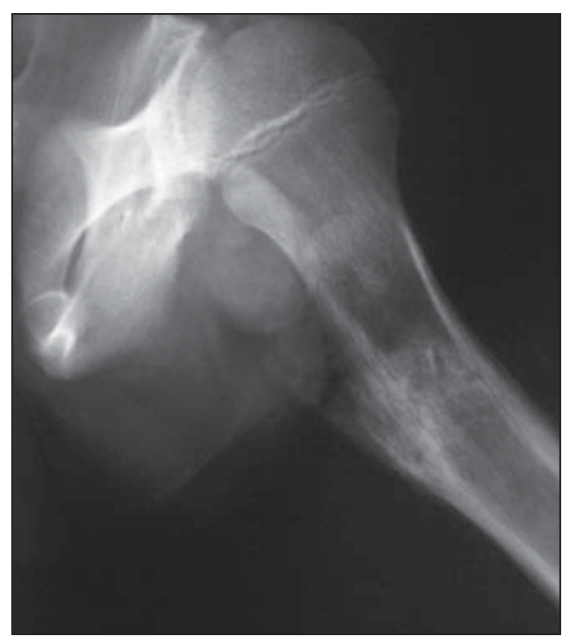

Figura 7. Sarcoma de Ewing do fêmur. Lesão metafisária proximal, lítica, com reação periosteal laminada interrompida, dando origem ao "triângulo de Codman".

descrição radiográfica do aspecto inicial e pré-tratamento em 22,1\% (50) dos casos, fato justificável, em parte, pois grande parte dos pacientes do estudo foi encaminhada em avançado estágio da doença e após algum tipo de tratamento. Entretanto, muitos prontuários eram preenchidos de forma incompleta.

Tratamento quimioterápico foi aplicado em $96 \%$ (216), tratamento cirúrgico em $70,3 \%$ (159) e tratamento radioterápico em $48,2 \%$ (109) dos pacientes.

\section{DISCUSSÃO}

O sarcoma de Ewing responde por cerca de $6 \%$ a $10 \%$ dos tumores ósseos malignos primários, sendo, portanto, o quarto mais frequiente deste grupo de lesões ${ }^{(\mathbf{1})}$. É um tumor de pequenas células redondas, bastante agressivo, muito semelhante, do ponto de vista histológico, a outras lesões como linfomas, neuroblastomas metastáticos e alguns tumores indiferenciados.

Muito se discute a respeito da verdadeira linhagem celular neoplásica desses sarcomas, entretanto, evidências recentes apontam para células de origem neuroectodérmica. De fato, estas mesmas evidências questionam se o sarcoma de Ewing e o tumor primitivo neuroectodérmico (TPNE) não são uma mesma entidade ${ }^{(2)}$. A translocação de uma porção do braço longo dos cromossomos 11 e 22 foi identificada nestas lesões ${ }^{(3)}$.
A distinção entre linfomas e o sarcoma de Ewing é geralmente simples, porém um grupo de lesões apresenta características próprias de cada uma delas, com células grandes e irregulares, sendo conhecido como sarcomas de Ewing atípicos ${ }^{(2)}$. Outra doença que pode simular a presença de um sarcoma de Ewing é a osteomielite; seu quadro clínico e os aspectos histológicos e radiológicos podem ser absolutamente similares. Um dado de valor na diferenciação radiológica de ambos é que na osteomielite comumente há perda da interface entre a gordura subcutânea e a musculatura adjacente, bem como obliteração dos planos de gordura intermusculares por edema secundário a inflamação local. O sarcoma de Ewing, por sua vez, raramente produz obliteração destes planos, mesmo com o grande aumento de partes moles quase sempre associado $^{(4)}$.

Existe também uma variante do sarcoma de Ewing que esporadicamente surge em partes moles, indistinguível histologicamente da lesão óssea. Ocorre mais comumente em jovens, na parede torácica, membros inferiores e regiões paravertebrais ${ }^{(\mathbf{5})}$.

Tipicamente, o sarcoma de Ewing ósseo acomete indivíduos na segunda década de vida, sendo que $75 \%$ apresentam menos de 20 anos de idade na época do diagnósti$\mathrm{co}^{(4)}$; raramente surge em indivíduos acima dos 50 anos de idade. Cerca de $66 \%$ dos pacientes são do sexo masculino.

O sintoma mais freqüente é dor, localizada ou generalizada, que progressivamente se torna intensa e persistente. Surge, amiúde, várias semanas ou meses antes de qualquer alteração radiológica ser identificada. Aumento de volume adjacente ao sítio da lesão é frequientemente relatado, porém raramente é a queixa inicial. Outros sintomas, mais raros, são derrame articular, febre, dificuldade de locomoção, anemia e perda de peso $^{(2,4)}$.

Qualquer osso do corpo pode ser afetado, porém os ossos das extremidades o são mais comumente; o acometimento dos ossos das mãos (Figura 8) e pés é raro ${ }^{(6,7)}$. A localização mais comum nos ossos longos é tema de divergência entre autores que revisaram grandes séries de pacientes com esta neoplasia $^{(\mathbf{2 , 4 , 8 )}}$. Na realidade, o tumor pode surgir em qualquer porção da medular de um osso tubular, principalmente nas 


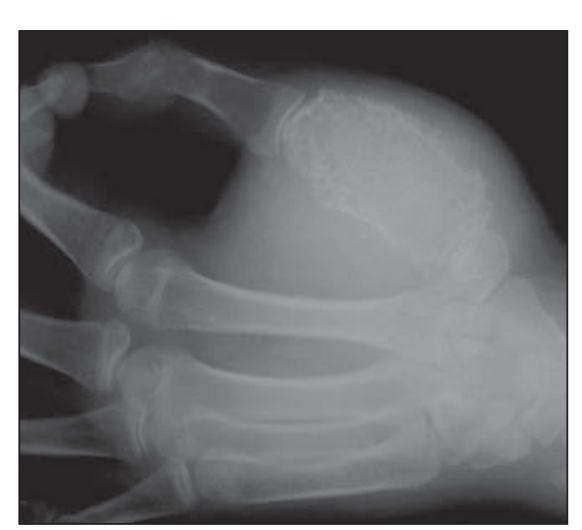

Figura 8. Sarcoma de Ewing do primeiro metacarpo. Os ossos das mãos e pés raramente são acometidos por esta entidade.

metáfises, entretanto, a diáfise também é comprometida com frequiência considerável. Tumores epifisários, apesar de muito raros, também são descritos ${ }^{(9)}$.

O sarcoma de Ewing é definido como periosteal quando seu componente subperiosteal não invade a cavidade medular ${ }^{(\mathbf{1 0})}$. É uma entidade rara, com apenas poucos casos descritos, que acomete predominantemente adolescentes do sexo masculino e raramente apresenta metástases na época do diagnóstico.

O aspecto radiográfico do sarcoma de Ewing é bastante variável, porém apresenta padrões mais prevalentes de acordo com o tipo de osso envolvido. Em ossos tubulares, o aspecto mais encontrado é o de lesão fusiforme, mal delimitada, permeativa, com aumento de partes moles e reação periosteal associada. Duas formas de reação periosteal são encontradas: uma é a laminar ou paralela (conhecida como padrão em "casca de cebola") e a outra é a perpendicular ou espiculada. Raramente a forma espiculada se apresenta isoladamente, sem um componente laminar. Nestes casos o diagnóstico diferencial com osteossarcoma se torna mais difícil. Incomum também é a forma esclerótica deste tumor, bem como a presença de calcificações de permeio. Nos ossos chatos, as lesões são tanto líticas quanto escleróticas e mistas ${ }^{(\mathbf{1 1})}$ e são similares a vários outros processos malignos, como o osteossarcoma, as metástases e lesões ósseas pseudotumorais e certas infecções. Portanto, nestes ossos, o padrão de imagem pouco auxilia no diagnóstico diferencial ${ }^{(\mathbf{9})}$.

Em cerca de 2\% dos sarcomas de Ewing o aspecto de imagem é absolutamente benigno $^{(\mathbf{1 2})}$. Existe inclusive um caso relatado de apresentação radiográfica simulando cisto ósseo solitário ${ }^{(\mathbf{1 3})}$. A tomografia computadorizada (TC) e a ressonância magnética (RM) são exames úteis no estadiamento destas lesões, definindo mais claramente as alterações da cortical, o componente de partes moles envolvido (musculatura e feixe neurovascular) e a presença de metástases na medular do mesmo osso.

Nas lesões de parede torácica, onde o sarcoma de Ewing apresenta-se como a mais comum lesão maligna em crianças, a TC é superior às radiografias simples na avaliação de presença e caracterização de uma lesão conhecida, bem como para determinar a característica lítica ou blástica da mesma, invasão da cavidade torácica e para eventualmente diferenciar derrames pleurais de massa de tecidos moles ${ }^{(\mathbf{1})}$.

Em exames de RM, o sarcoma de Ewing demonstra sinal de baixa intensidade em sequiências ponderadas em T1 e sinal elevado em seqüências ponderadas em T2, STIR e T2*(14) , com definição excelente da extensão da lesão na medular óssea e do edema peritumoral.

Com o surgimento de múltiplos e novos agentes quimioterápicos, o prognóstico desta classe de tumores mudou sensivelmente. Hoje, a sobrevida em dez anos varia de $50 \%$ a $70 \%{ }^{(15)}$ e a $\mathrm{RM}$ tem papel importante na monitoração da resposta à quimioterapia $^{(\mathbf{1 6})}$. Nos tumores em que a cirurgia pode ser realizada com margens seguras, pode se abrir mão de radioterapia pós-operatória e o prognóstico de sobrevida em dez anos sobe para $75 \%{ }^{(\mathbf{1 7})}$. Por sua vez, os tumores da coluna ou aqueles muito volumosos na pelve, geralmente inoperáveis, apresentam prognóstico sombrio e a radioterapia é usada de forma adjuvante, em conjunto com a quimioterapia.

\section{REFERÊNCIAS}

1. Coombs RJ, Bayar EA, Matloub YH, Velasco ME. Pediatric case of the day. Ewing sarcoma of the rib with intraspinal extension. RadioGraphics 1999; 19:241-244.

2. Unni KK. Dahlin's bone tumors. 5th ed. Philadelphia: Lippincott-Raven, 1996;249-261.

3. Unni KK, Inwards CY. Tumors of the osteoarticular system. In: Fletcher CDM, editor. Diagnostic histopathology of tumors. New York: Churchill Livingstone, 1995;1104-1106.

4. Mirra JM. Bone tumors: diagnosis and treatment. 2nd ed. Philadelphia: JB Lippincott, 1980.

5. Cheung CC, Kandel RA, Bell RS, Mathews RE, Ghazarian D. Extraeskeletal Ewing sarcoma in a 77-year-old woman. Arch Pathol Lab Med 2001; 125:1358-1360.

6. Yamaguchi T, Tamai K, Saotome K, Hoshino T, Masawa N. Ewing's sarcoma of the thumb. Skeletal Radiol 1997;26:725-728.

7. Baraga JJ, Amrami KK, Swee RG, Wold L, Unni KK. Radiographic features of Ewing's sarcoma of the bones of the hands and feet. Skeletal Radiol 2001;30:121-126.

8. Dahlin DC, Coventry MB, Scanlon PW. Ewing's sarcoma: a critical analysis of 165 cases. Am J Orthop 1961;43A:185-192.

9. Sherman RS, Soong KY. Ewing's sarcoma: its Roentgen classification and diagnosis. Radiology 1956;66:529-539.

10. Shapeero LG, Vanel D, Sundaram M, et al. Periosteal Ewing sarcoma. Radiology 1994;191:825831.

11. Baker ND, Dorfman DM. Ewing's sarcoma of the sacrum. Skeletal Radiol 1996;25:302-304.

12. Netherlands Committee on Bone Tumors. Radiological atlas of bone tumors. Amsterdam: Elsevier, 1993;193-214,579-590.

13. Bhagia SM, Grimer RJ, Davies AM, Mangham DC Ewing's sarcoma presenting as a solitary bone cyst. Skeletal Radiol 1997;26:722-724.

14. Boyko OB, Cory DA, Cohen MD, Provisor A, Mirkin D, DeRosa GP. MR imaging of osteogenic and Ewing's sarcoma. AJR 1987;148:317-322.

15. Wilkins RM, Pritchard DJ, Burgert EO Jr, Unni KK. Ewing's sarcoma of bone. Experience with 140 patients. Cancer 1986;58:2551-2555.

16. Rosen G, Caparros B, Nirenberg A, et al. Ewing's sarcoma: ten-year experience with adjuvant chemotherapy. Cancer 1981;47:2204-2213.

17. Stoller DN, Johnson JO, Steinkirchner TM. Tumores do osso e tecidos moles. In: Stoller DN, editor. Ressonância magnética em ortopedia \& medicina desportiva. $2^{\underline{a}}$ ed. Rio de Janeiro: Guanabara Koogan, 1997;1228-1230. 